\title{
Einsatz von Telemedizin in der Palliative Care
}

\author{
Andreas Samuel Ebneter ${ }^{a}$, Thomas Sauter ${ }^{b}$, Annika Achter ${ }^{\text {, }}$, Steffen Eychmüller ${ }^{\mathrm{d}}$ \\ a Dr. med., Universitäres Zentrum für Palliative Care, Inselspital Bern, Bern; b Prof. Dr. med., Universitäres Notfallzentrum, Inselspital Bern, Bern und \\ Telenotfallmedizin, Universität Bern; ${ }^{c}$ Dr. med., Permanence H-FR Meyriez-Murten, H-FR, Meyriez; ${ }^{d}$ Prof. Dr. med., Universitäres Zentrum für Palliative Care, \\ Inselspital Bern, Bern
}

Wir leben in einer komplexen Welt mit komplexen gesundheitlichen Situationen, welche nur mit der Hilfe von interprofessioneller Koordination bewältigt werden können. Die Telemedizin als digitale Kommunikationsform kann helfen, die interprofessionelle Zusammenarbeit zu verbessern. Sie vereinfacht den Austausch im Team sowie mit den Patientinnen und Patienten und deren Angehörigen. Das zeigt sich exemplarisch beim Einsatz der Telemedizin in der Palliative Care.

In einer Welt mit immer komplexeren gesundheitlichen Situationen ist die Interprofessionalität ein wichtiges Element der medizinischen Teamarbeit [1]. Interprofessionalität überspannt nicht nur Fachgebiete und Berufsgruppen, sondern auch verschiedene Sektoren des Gesundheitswesens vom Spital über die ambulante Betreuung bis zur Langzeitpflege.

Die Definition von interprofessioneller Zusammenarbeit ist uneinheitlich [1, 2]. Allen Definitionen gemeinsam ist der Fokus aller beteiligten Berufsgruppen auf ein bestimmtes, gemeinsames Ziel. Davon zu unterscheiden ist die multiprofessionelle Zusammenarbeit, in welcher jede Berufsgruppe die eigenen Ziele verfolgt.

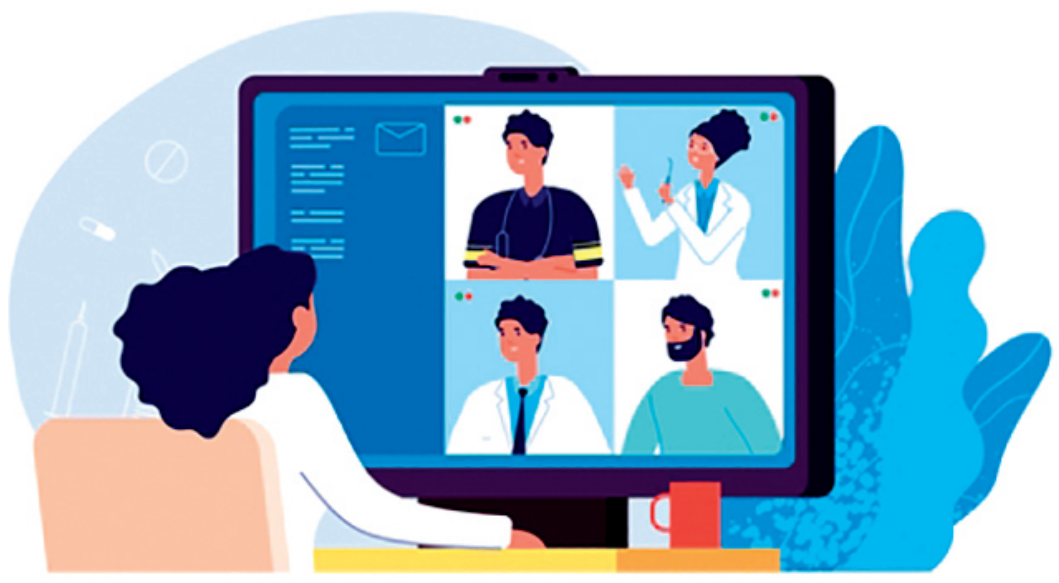

Die Telemedizin kann viel zur Kommunikation und Koordination in der interprofessionellen Zusammenarbeit beitragen.

\section{Dynamische Kommunikationsform}

Das gemeinsame Ziel ist der wichtigste Aspekt der Interprofessionalität. Die Telemedizin kann einen wichtigen Beitrag dazu leisten, diese patientenzentrierten Ziele in einem partizipativen Vorgehen zu definieren. Die Telemedizin als digitale Gesundheitsintervention ist eine dynamische Kommunikationsform und kann ideal zur Kommunikation und Koordination insbesondere in der interprofessionellen Zusammenarbeit eingesetzt werden. Das elektronische Patientendossier ist im Unterschied dazu eine statische Sammlung von Dokumenten [3].

Verschiedene Studien zur Anwendung der Telemedizin existieren bereits in der Palliative Care [4], bei multimorbiden Patientinnen und Patienten [5, 6], in der Langzeitpflege [7] sowie in der Neurologie und Rehabilitation [8]. Als positiver Effekt zeigt sich in diesen Studien eine Verbesserung der Teamkoordination und -effizienz. Im Langzeitbereich zeigt sich zusätzlich ein Qualitätsgewinn durch den schnelleren Zugang zu Fachspezialistinnen und -spezialisten [7].

\section{Telemedizin in der Palliative Care}

Die Telemedizin ist in der Palliative Care, wenn geographische oder andere Barrieren (z.B. Covid-19) bestehen, gut etabliert [9]. Das häufigste Anwendungsfeld ist die virtuelle Visite, also eine Videokonsultation zwischen einer Fachperson und der betroffenen Person oder deren Angehörigen. Trotz der Ausrichtung 
auf eine direkte und persönliche Beziehung ist die virtuelle Visite in der Palliative Care grundsätzlich gut möglich und akzeptiert, und sie zeigt Vorteile in der Kommunikation, Koordination und Stressreduktion bei den Patientinnen und Patienten sowie den Angehörigen $[9,10]$. Studien aus Ländern mit einer hohen Ärztedichte und geographisch eher engen Verhältnissen wie der Schweiz fehlen aber bisher [10].

Die virtuelle Visite ist eine bilaterale Form der Kommunikation am Bildschirm. Diese unterscheidet sich von der interprofessionellen Besprechung, welche zwei Teilnehmerkreise haben kann: a) nur Fachpersonen («TEAM/Care-Konferenz», [Tumor-]Boards) oder b) Fachpersonen und Betroffene/Angehörige mit einer aktiven Rolle der Patientinnen und Patienten und der Angehörigen («Rundtischgespräche»).

\section{Digitale interprofessionelle Besprechung}

Im Kontext der Behandlung von komplexen, polymorbiden Patientinnen und Patienten und bei der Palliative Care existieren bis jetzt vor allem Care-Konferenzen. Hier zwei Beispiele:

Für die Behandlung von polymorbiden Personen hat die Auswertung einer strukturierten interprofessionellen Besprechung im Sinn eines Rundtischgesprächs

In interprofessionellen Besprechungen können Wissen, Erfahrung und praktische Fähigkeiten ausgetauscht werden.

zwischen Allgemeinärztin resp. -arzt, Patientin resp. Patient und Pflege einerseits und einem Team von verschiedenen Fachpersonen andererseits Vorteile gezeigt: Wissen, Erfahrung und praktische Fähigkeiten können gut ausgetauscht werden. Neben den gemeinsamen Zielen werden realitätsnahe und unmittelbar umsetzbare Informationen weitergegeben. Die traditionelle Konsultation wird so zu einer interprofessionellen Plattform der Zusammenarbeit.

Nicht zu vernachlässigen ist der Lerneffekt für alle Beteiligten [5, 6]. Finanziell zeigen sich ebenfalls Vor-

\section{Projekt am Inselspital Bern}

Am Inselspital wurde in einer Kooperation der Palliativmedizin und der Telenotfallmedizin an der Universität Bern das epall.ch Projekt (Telemedizin in der Palliative Care) initiiert. Ziel dieses Projektes ist die Beantwortung der Frage, wie die Telemedizin in die komplexe Behandlung von Palliative-Care-Patientinnen und -Patienten integriert werden kann und auf welcher Ebene die Telemedizin hilfreich für die Betroffenen, ihre Angehörigen und das interprofessionelle Team sein kann. Das Projekt kann unter www.epall.ch verfolgt werden.

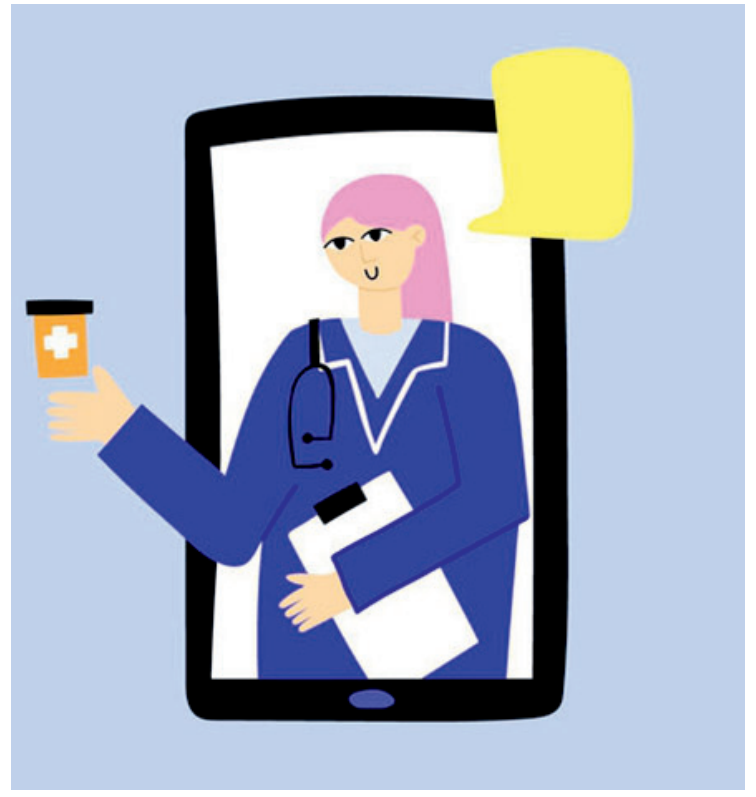

In der Palliative Care kommt die Telemedizin am häufigsten in Form von virtuellen Visiten zum Einsatz.

teile: Komplexe Situationen interprofessionell zu besprechen und einen gemeinsamen Plan zu erstellen ist kostengünstiger als ein Hospitalisationstag [6]. Als Nachteil dieser Besprechungsform wurde die zeitintensive Vor- und Nachbereitung eingestuft.

In einem kanadischen Modell wurde im Rahmen eines virtuellen Schmerz- und Symptomkontroll-Programmes der Palliative Care ein Online-Rundtischgespräch zwischen einem Patienten, Angehörigen, einem Palliativmediziner sowie weiteren Fachpersonen aus dem interprofessionellen Team organisiert. Vorteile zeigten sich patientenseitig in einer Symptomreduktion (Angst und Appetitlosigkeit) sowie in einer reduzierten Reisezeit und geringeren Kosten. Als Nachteil zeigte sich aus Sicht der Fachpersonen wiederum die aufwendige und komplexe Organisation im Vorfeld [11]. Zeitmangel und mangelnde Flexibilität sind auch in einer anderen Studie teilweise ein Hindernis für interprofessionelle Besprechungen, obwohl der zu erwartende Nutzen positiv eingeschätzt wird [4].

\section{Zur Umsetzung in der Schweiz}

Gute Kommunikation und Koordination ist auch im schweizerischen Gesundheitssystem mit der Fragmentierung der Gesundheitssektoren und der hohen Dichte an Fachspezialistinnen und -spezialisten essentiell. Die Telemedizin, insbesondere in Form von digitalen Rundtischgesprächen, kann hier helfen. Für einen routinemässigen Einsatz im interprofessionellen Kontext bedarf es der besonderen Anstrengung, diese Hilfsmittel datenschutzkonform und einfach für 
alle Fachpersonen, Patientinnen und Patienten sowie Angehörigen zugänglich zu machen.

Die technologischen Voraussetzungen sind in der Schweiz gegeben. Die wichtigsten elektronischen Tools funktionieren und genügen den Anforderungen von Daten- und Persönlichkeitsschutz [12]. Die Erfahrung mit digitalen Hilfsmitteln ist in der Schweiz noch beschränkt und diese werden bis jetzt nur limitiert eingesetzt und genutzt [13]. Auch wird im Medizinstudium der Einsatz digitaler Hilfsmittel bisher nur selten vermittelt [14]. Ob und in welchem Umfang sich die Telemedizin der Zukunft etablieren wird, ist noch unklar. Die Corona-Pandemie hat jedoch viele Zielgruppen für mögliche Anwendungen der Telemedizin im Schweizer Gesundheitssystem sensibilisiert.

Verschiedene Faktoren werden für eine erfolgreiche Umsetzung der Telemedizin mitverantwortlich sein. Es muss sich erst zeigen, ob die Erfahrungen aus anderen Ländern mit sehr grossen geographischen Entfernungen zwischen Fachpersonen und Patientinnen und Patienten und Angehörigen auf die Schweiz mit einer vergleichsweise hohen Ärztedichte sowohl in den städtischen Gebieten wie auch den ländlichen Gebieten übertragen werden können. Zu unterscheiden ist wohl auch der Einsatz der Telemedizin einerseits in der Grundversorgung [15] und andererseits in der Notfallversorgung [16]

Die Telemedizin bietet nicht zuletzt in Zeiten der Corona-Pandemie Chancen für eine verbesserte Kommunikation und Koordination. Auch zeigt sich, dass gerade in einer auf die persönliche Kommunikation ausgerichteten Disziplin wie der Palliative Care die Nutzung von digitalen Hilfsmitteln in der interprofessionellen Zusammenarbeit Vorteile haben könnte, insbesondere innerhalb der regionalen medizinischen Netzwerke.

Unsere Zukunft wird digital sein, nutzen wir also die Vorteile der Digitalisierung dafür, uns für das Patientenwohl zu vernetzen: interprofessionell und patientenzentriert.

\section{Danksagung}

Das Universitäre Notfallzentrum dankt dem Touring Club Schweiz für die Unterstützung der Telenotfallmedizin an der Universität Bern.

\section{Bildnachweis}

Microvone | Dreamstime.com

Maria Lutskovskaya | Dreamstime.com

\section{Schwerpunktserie Interprofessionalität}

Die interprofessionelle Zusammenarbeit von Fachpersonen aus verschiedenen Gesundheitsberufen gilt als wichtiges Mittel, um den Herausforderungen im Gesundheitswesen zu begegnen. Aber wie weit ist die Schweiz in diesem Bereich tatsächlich? Welche Hürden und Chancen gibt es? In unserer Schwerpunktserie betrachten wir das Thema aus unterschiedlichen Perspektiven.

\section{Literatur}

1 Schmitz C, Atzeni G, Berchtold P. Wieso eigentlich interprofessionelle Zusammenarbeit. Schweiz Ärzteztg. 2020;101(9):292-3.

2 Mahler C, Gutmann T, Karstens S, Joos S. Terminology for interprofessional collaboration: definition and current practice. GMS Z Med Ausbild. 2014;31(4):Doc40.

3 WHO. WHO | Classification of digital health interventions v1.0. [Available from:] http://www.who.int/reproductivehealth/ publications/mhealth/classification-digital-health-interventions/en/

4 van Gurp J, van Selm M, van Leeuwen E, Vissers K, Hasselaar J. Teleconsultation for integrated palliative care at home: A qualitative study. Palliative medicine. 2016;30(3):257-69.

5 Boeckxstaens P, Brown JB, Reichert SM, Smith CNC, Stewart M, Fortin M. Perspectives of specialists and family physicians in interprofessional teams in caring for patients with multimorbidity: a qualitative study. CMAJ Open. 2020;8(2):E251-E256.

6 Pariser P, Pham TT, Brown JB, Stewart M, Charles J. Connecting People With Multimorbidity to Interprofessional Teams Using Telemedicine. Ann Fam Med. 2019;17(Suppl 1):S57-S62.

7 Tan AJ, Rusli KD, McKenna L, Tan LL, Liaw SY. Telemedicine experiences and perspectives of healthcare providers in long-term care: A scoping review. J Telemed Telecare. 2021:1357633x211049206.

8 Careau E, Vincent C, Noreau L. Assessing interprofessional teamwork in a videoconference-based telerehabilitation setting. J Telemed Telecare. 2008;14(8):427-34.

9 Finucane AM, O’Donnell H, Lugton J, Gibson-Watt T, Swenson C, Pagliari C. Digital health interventions in palliative care: a systematic meta-review. NPJ Digit Med. 2021;4(1):64.

10 Ebneter AS, Fliedner M, Trapp D, Ramseier F, Sauter TC, Eychmüller S. Telemedizin in der Palliative Care: Digitale Kommunikation in einem beziehungsbasierten Fachgebiet - ist das sinnvoll? Praxis. 2021;110(15):845-50.

11 Watanabe SM, Fairchild A, Pituskin E, Borgersen P, Hanson J, Fassbender K. Improving access to specialist multidisciplinary palliative care consultation for rural cancer patients by videoconferencing: report of a pilot project. Support Care Cancer. 2013;21(4):1201-7.

12 FMH. Telemedizin 2021. [Available from:] https://www.fmh.ch/ themen/ehealth/telemedizin.cfm

13 Mettler AC, Piazza L, Michel J, Muller M, Exadaktylos AK, Hautz WE, et al. Use of telehealth and outcomes before a visit to the emergency department: a cross-sectional study on walk-in patients in Switzerland. Swiss Med Wkly. 2021;151:w20543.

14 Hautz SC, Hoffmann M, Exadaktylos AK, Hautz WE, Sauter TC. Digital competencies in medical education in Switzerland: an overview of the current situation. GMS J Med Educ. 2020;37(6):Doc62.

15 Stücheli B. Als Hausarzt leiste ich Notfalldienst zumindest im Moment noch. Schweiz Ärzteztg. 2019;100(29-30):964-5.

16 Sauter TC, Hautz WE, Lehmann B, Exadaktylos AK. eNotfallmedizin: Notfallversorgung der Zukunft. Schweiz Ärzteztg. 2019;100(49):1683-5. 